\title{
Fluoxetine reduced depressive symptoms and alcohol consumption in patients with comorbid major depression and alcohol dependence
}

\author{
Cornelius JR, Salloum IM, Ehler JG, et al. Fluoxetine in depressed alcoholics. A double-blind, placebo-controlled trial. Arch Gen \\ Psychiatry 1997 Aug;54:700-5.
}

\section{Objective}

To evaluate the effectiveness of fluoxetine in reducing depressive symptoms in patients with comorbid major depression and alcohol dependence.

\section{Design}

Randomised, double blind, placebo controlled, parallel group trial with 12 weeks of follow up.

\section{Setting}

Western Psychiatric Institute and Clinic of the University of Pittsburgh, Pennsylvania, USA.

\section{Patients}

51 patients between 18 and 65 years of age (mean age $35 \mathrm{y}$, $51 \%$ men) diagnosed with current comorbid major depressive disorder and alcohol dependence using the Structured Clinical Interview for Diagnostic and Statistical Manual of Mental Disorders, 3 rd edition, revised criteria. Exclusion criteria were a diagnosis of bipolar disorder; schizoaffective disorder; schizophrenia; non-alcohol substance dependence; hyperthyroidism or hypothyroidism; liver disease (liver function test $\geqslant 3$ times normal); cardiac or renal impairment; pregnancy; mental retardation; clinically evident cognitive impairment; or antipsychotic or antidepressant medication use in the previous month. $90 \%$ completed the study.

\section{Intervention}

25 patients were allocated to fluoxetine, initially given one 20 mg capsule that could be increased to 2 capsules after 2 weeks if substantial residual depressive symptoms persisted. 26 patients were allocated to identical placebo. Patient randomisation was stratified by sex and race. All patients also received usual care for dual diagnosis patients consisting of weekly psychotherapy sessions and weekly meetings with a psychiatrist.

\section{Main outcome measures}

Weekly ratings of depression using the 24 item Hamilton Rating Scale for Depression (HAM-D-24), the Beck Depression Inventory (BDI), and the Global Assessment Scale (GAS); and weekly ratings of drinking using a timeline follow back method and the Addiction Severity Index.

\begin{abstract}
Main results
Change in the HAM-D-24 (improvement) was greater in the fluoxetine group than in the placebo group $(-6 v-2$, $\mathrm{p}<0.05)$. No difference existed between the groups for change in the BDI $(p=0.17)$. Improvement in the mean GAS score was greater in the fluoxetine group compared with the placebo group $(17 v 5, \mathrm{p}=0.005)$. The mean total number of drinks consumed during the 12 week trial was lower in the fluoxetine group than in the placebo group $(70 v 216$, $\mathrm{p}<0.03)$ as was the mean number of days on which alcoholic drinks were consumed during the trial $(11 v 20, \mathrm{p}<0.05)$. The total number of heavy drinking days (defined as $\geqslant 5$ drinks per day) was also lower in the fluoxetine group ( $5 v 16, \mathrm{p}=0.04)$. The number of weeks until first heavy drinking was higher in the fluoxetine group ( $8 v 5, \mathrm{p}=0.02$ ) but proportion of patients completely abstinent during the trial did not differ $(28 \%$ v $15 \%, \mathrm{p}=0.27)$. Fluoxetine was well tolerated.
\end{abstract}

\section{Conclusion}

Fluoxetine reduced depressive symptoms and alcohol consumption in patients with comorbid major depression and alcohol dependence at 12 weeks.

Sources of funding: National Institute on Alcohol Abuse and Alcoholism and Mental Health Clinical Research Center.

For article reprint:Dr J R Cornelius, Western Psychiatric Institute and Clinic, 3811 O'Hara Street, Room 1092, Pittsburgh, PA 15213, USA. Fax +1 4126244699.

\section{Commentary}

This is the first double blind, placebo controlled study to show the effectiveness of a selective serotonergic drug in the reduction of depressive symptoms and alcohol consumption in patients with comorbid major depressive and alcohol dependence disorders. A previous trial evaluating fluoxetine found no effect on alcohol consumption, but fluoxetine did reduce Ham-D-24 scores more than placebo treatment among the $14 \%$ of study subjects who had comorbid depression.

In this study by Cornelius et al, the reduction in Ham-D-24 score in the fluoxetine group after detoxification and washout from 19 to 13 , compared with 18 to 16 in the placebo group, would have added clinical significance if this reduction was associated with a concomitant reduction in current suicidal ideation. Unfortunately, these data were not reported.

Inclusion in the study required a primary major depressive disorder diagnosis. The $44 \%$ reduction in Ham-D-24 scores between presentation and conclusion of detoxification and washout in this study is similar to that reported by Brown et al in patients with comorbid primary alcohol dependence and secondary depressive disorder. ${ }^{2}$ Thus, application of the results found by Cornelius et al to this clinical population may be appropriate.

On presentation, the patients in this study were severely depressed. After detoxification and washout patients in both groups appeared to have been moderately depressed based on depression outcome measure scores. It may be reasonable therefore to apply these results to patients with alcoholism who have mod- erate, rather than severe, depression after detoxification and washout.

Beginning fluoxetine treatment after a 2-4 week period of abstinence, compared with 1 week in this study, may be more appropriate (and is probably more common) in clinical practice. Fluoxetine treatment of patients with clinically significant liver disease requires caution and possibly $a \geqslant 50 \%$ dosage reduction.

David RS Haslam, MSc, MD Dalhousie University Halifax, Nova Scotia, Canada

1 Kranzler HR, Burleson JA, Korner P, et al. Am J Psychiatry 1995;152:391-7.

2 Brown SA, Inaba RK, Gillin JC, et al. Am J Psychiatry 1995;152:45-52.

Schenker S, Bergstrom RF, et al. Clin Pharmacol Ther 1988;44:353-9.
Then 